\title{
Fusion - fission dynamics: fragment mass distribution studies
}

\author{
S. Bhattacharya ${ }^{1, a}$, A. Chaudhuri ${ }^{1}$, T.K.. Ghosh ${ }^{1}$, K. Banerjee ${ }^{1}$, C. Bhattacharya ${ }^{1}$, S.Kundu $^{1}$, G. Mukherjee ${ }^{1}$, T. K. Rana ${ }^{1}$, P. \\ Roy $^{1}$, R. Pandey ${ }^{1}$, P. Bhattacharya ${ }^{2}$ \\ ${ }^{1}$ Variable Energy Cyclotron Centre, 1/AF Bidhan Nagar, Kolkata 700 064, India \\ ${ }^{2}$ Saha Institute of Nuclear Physics, 1/AF Bidhan Nagar, Kolkata 700 064, India
}

\begin{abstract}
Using the major accelerator facilities available in India, detailed experimental studies have been made to understand the mechanism of quasi-fission and role of nuclear shell effect in heavy nuclei. Fission fragment mass distribution has been used as the probe to explore the role of entrance channel effects on fusion-fission and quasifission dynamics. Fission fragment mass distribution has also been demonstrated to be useful to identify the phenomenon of 'washing out' of nuclear shell effect with excitation energy.
\end{abstract}

\section{Introduction}

Recently, there has been a lot of interest in the study of the mechanism of fusion reaction at near-barrier energies in the actinide region, which plays a key role in the synthesis of super heavy elements (SHE) [1-3]. The formation of SHE, through the fusion of two nuclei, requires that the target and projectile must have enough kinetic energy to overcome the repulsive electrostatic (Coulomb) barrier in order to come within the range of the attractive nuclear forces in a touching configuration. Since the probability of decay of a compound nucleus increases with excitation energy, it is desirable that compound nucleus (or SHE) is produced at moderate excitation energy, i.e., near the Coulomb barrier energy where fusion cross section is not very low.

In the fusion of two nuclei near Coulomb barrier energy, the reaction dynamics is mainly governed by the interplay of three processes;

(a) After surmounting the Coulomb barrier, the reactants get trapped in an attractive potential pocket and produce a composite di-nuclear system, which gradually evolves to form a compact mono-nuclear configuration (compound nucleus) after equilibration in all (energy, mass and shape) degrees of freedom. The excited compound nucleus $(\mathrm{CN})$ may then end up as an evaporation residue (ER) after emitting neutrons, light charged particles and gamma rays.

(b) If the compound nucleus is of higher fissility and produced at higher excitation energy, it reaches the unconditional mass-symmetric saddle through shape oscillations and subsequently undergoes binary fission. This is known as compound nuclear fission or fusionfission (FF).

(c) However, in some of the cases, it is found that the composite system breaks up in fission like events before achieving complete equilibration in shape and mass degrees of freedom. This is known as quasi-fission (QF). Other incomplete fission process like fast fission dominates at higher excitation energies where the angular momentum dependent fusion barrier vanishes. At moderate excitation energies, fast fission is negligible. Quasi-fission is a serious competitor for the formation of a compound nucleus and subsequently, the formation of an ER. Since quasi-fission occurs before the target and the projectile fuse to form a compound nucleus, it hinders the formation of the ER. It is a prominent reaction channel at low excitation energies, just above the fusion threshold, where the ER formation is also dominant. Hence, for a proper choice of target-projectile combination aiming at the synthesis of a super heavy element, it is important to understand the reaction dynamics of fusion-fission and quasi-fission at beam energies close to the Coulomb barrier for different targetprojectile combinations of varying entrance channel mass asymmetry.

In a series of experiments carried out at the major Indian accelerator facilities, we have studied fusion-fission visà-vis quasi-fission processes at beam energies close to the Coulomb barrier for different target projectile combinations. Here we present a review of our findings on the roles of target deformation and entrance channel mass asymmetry on fusion-fission and quasi-fission processes using fission fragment mass distribution probe. We further report our new result on the use of mass distribution as a direct probe for 'observing' washing out of shell effect with excitation energy.

\section{Fusion-Fission versus Quasi-Fission}

Quasi-fission is, in many ways, similar to fusion-fission. In the case of fusion-fission, as the two nuclei collide,

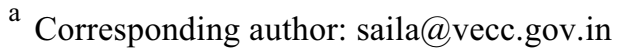


fusion occurs when the system overcomes the internal frictional energy loss, and gradually evolve from a contact configuration to a conditional saddle configuration. This configuration can be defined as being the one corresponding to the maximum of potential energy with the initial value of mass asymmetry partially relaxed. Then the frictional forces trap the system and a mono-nuclear system is formed. Depending on the potential landscape, the system may evolve further to reach the unconditional saddle configuration with no constrain on the mass asymmetry, shape and $\mathrm{K}$ degrees of freedom, leading to symmetric mass splitting of the composite (fusion-fission).

The compound nuclear fission or fusion fission (FF) mechanism is illustrated in Fig.1 (upper panel) using a schematic, one-dimensional landscape of interaction potential [2] as a function of the inter-nuclear distance $R$. In the interaction regime, a neck is formed between two nuclei facilitating exchange of nucleons between the two and a single composite system is formed with two mass and charge distribution centres. This initial fusion stage is visualised in the sudden approximation, where the density of the composite system is assumed to remain identical to that of ions at large distance, i.e; the density remains frozen throughout this stage. The interaction potential obtained using frozen densities is termed as sudden potential. However, the potential landscape becomes more complicated during the subsequent evolution of the fused system. The collective excitations of the shape and mass degrees of freedom transform the potential energy landscape into a multidimensional one where shape and mass degrees of freedom relax to equilibrium. The interaction potential thus obtained is termed as adiabatic potential. From Fig. 1 (upper panel), it is evident that the system is trapped in the entrance channel after surmounting the fusion barrier. The saddle configuration is seen to be elongated enough to keep the system trapped and the system finally evolves to the formation of compound nucleus. Then the compound nucleus ends up in fission by crossing the fission barrier (unconditional saddle point).

The quasi-fission (QF) process is, on the other hand, characterized by full energy relaxation but incomplete relaxation of mass and shape degrees of freedom. The QF process may be explained following Swiatecki's dynamical model $[4,5]$. It was assumed that dynamical evolution of the two colliding nuclei could be described by a sequence of shapes of two spheres connected by conical neck. The complete specification of the system, formed after the collision of the target and the projectile, needs three microscopic variables: the first is related to the distance separating the centre of mass of each nucleus or to the elongation of the system when the two fragments strongly overlap, the second is associated with the mass asymmetry and the third is related to the properties of neck.

The mechanism of quasi-fission is illustrated in Fig. 1 (lower panel) in the landscape of interaction potentials as a function of inter-nuclear distance R. A typical trajectory of the system leading to QF is shown in figure by the arrow marked curve. Here, the system reaches mass asymmetric saddle, but not the mass symmetric unconditional saddle, which is more compact. So, the system fuses and fission occurs without passing through the fully equilibrated compound nuclear configuration. Thus the fused system is restricted by a mass asymmetric fission barrier - leading to incomplete equilibration of mass and shape degrees of freedom.

In general, for heavy systems (typically, $Z_{T} Z_{P}>1600$, where $Z_{T}$ and $Z_{P}$ are the target and projectile atomic numbers), the compactness of the exit channel configuration causes hindrance to the formation of a mono-nucleus leading to quasi-fission. However, the existence of quasi-fission has also been demonstrated for much lighter systems $\left(\mathrm{Z}_{\mathrm{T}} \mathrm{Z}_{\mathrm{P}} \sim 800\right)$ [6].

In recent times, various experimental observables like charge mass and kinetic energies of fission fragments have been quite successfully described using macroscopic approaches wherein the dynamic evolution of the target projectile di-nuclear system was described considering coupling of deep inelastic scattering, quasi-fission and fusion fission channels. [7-10]. Quasi-fission has also been quite successfully studied using time-dependent Hartree-Fock theory which is a fully microscopic quantum many body approach [11-12].

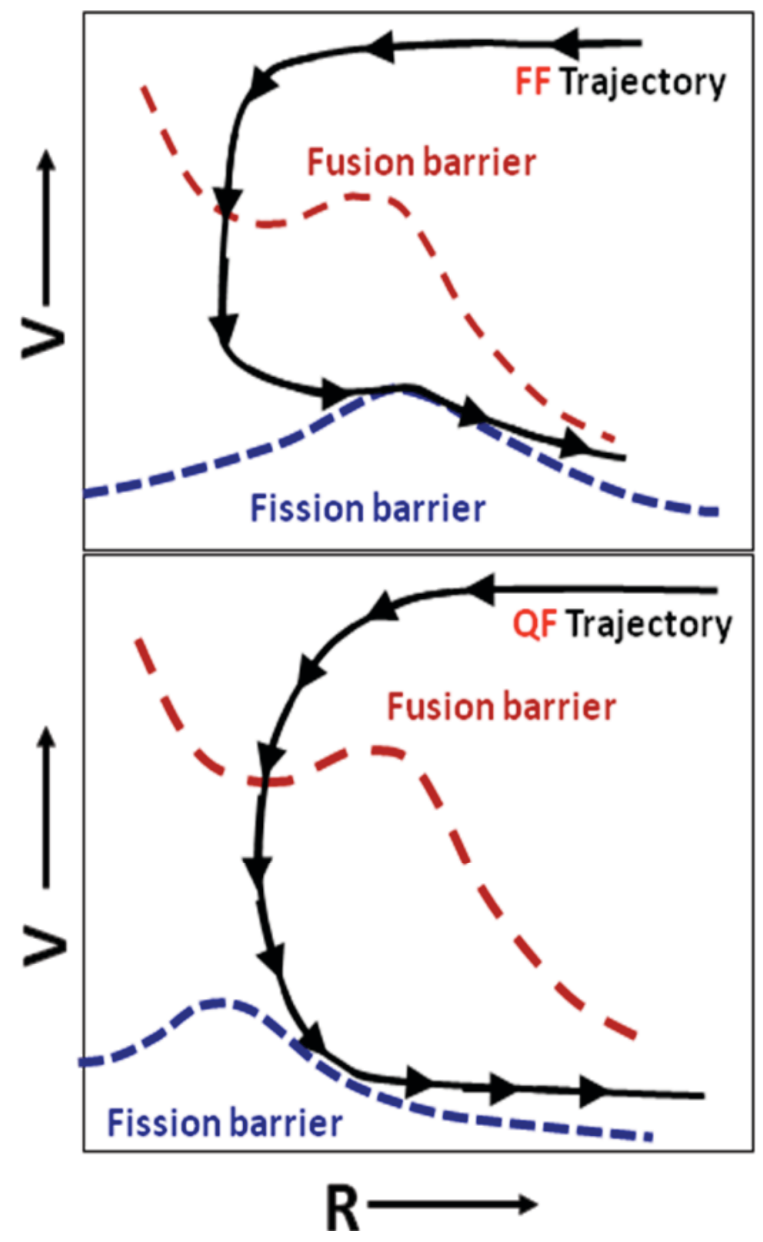

Figure 1. The potential energy diagrams for fusion-fission and quasi-fission processes. 


\section{Probes for Quasi-Fission}

Several experimental tools are now available to look for the existence of quasi-fission in a reaction. These involve measurement of (a) evaporation residue cross section, (b) mass-angular distribution of the fission fragments and (c) pre-scission neutron multiplicity.

\subsection{Measurement of ER Cross-section}

Evaporation residue cross-section is generally assumed to be a culmination of three factors which can be mathematically represented as:

$$
\sigma_{\mathrm{ER}}\left(\mathrm{E}_{\mathrm{cm}}\right)=\sigma_{\text {capture }}\left(\mathrm{E}_{\mathrm{cm}}\right) \cdot \mathrm{P}_{\mathrm{CN}}\left(\mathrm{E}_{\mathrm{cm}}\right) \cdot \mathrm{P}_{\text {survival }}\left(\mathrm{E}_{\mathrm{cm}}\right) \text {, }
$$

where $\sigma_{\text {capture }}$ is the capture cross-section for the formation of the di-nucleus system and $\mathrm{E}_{\mathrm{cm}}$ is the entrance channel kinetic energy of the system in the centre of mass. $\mathrm{P}_{\mathrm{CN}}$ is the probability of complete fusion of the dinuclear system to form the compound nuceus, which means complete equilibration in all degrees of freedom of the initial dinuclear system after capture. $P_{\text {survival }}$ is the survival probability of the $\mathrm{CN}$ to form $\mathrm{ER}$ at a given excitation energy which is determined by the competition between fission and evaporation of the excited compound nucleus. In systems prone to quasi-fission, the probability of complete fusion $\left(\mathrm{P}_{\mathrm{CN}}\right)$ is reduced considerably and this results in suppression of ER cross-section. This suppression of measured ER cross-section (in comparison with respective statistical model calculation) is treated as an evidence of quasi-fission.

\subsection{Measurement of FF Angular Anisotropy}

Fission fragment angular anisotropy (A) is defined as the ratio of the fragments yields emitted at the beam direction to the perpendicular direction $\left[\mathrm{W}\left(0^{\circ}\right) / \mathrm{W}\left(90^{\circ}\right)\right]$. In the statistical saddle point model, angular anisotropy is expressed as

$$
\mathrm{A}=1+<1^{2}>/ 4 \mathrm{~K}_{0}^{2}
$$

where, $\left\langle 1^{2}>\right.$ is the second moment of the compound nucleus spin distribution. The variance of the $K$ (projection of total angular momentum on fission axis) distribution, $\mathrm{K}_{0}{ }^{2}$, is given as $\mathrm{I}_{\text {eff }} \mathrm{T} / \mathrm{h}^{2}$, where $\mathrm{I}_{\text {eff }}$ is the effective moment of inertia and $\mathrm{T}$ is the temperature at the saddle point.

As the mass asymmetry degree of freedom may not be equilibrated in quasi-fission reactions and the fact that the mass-asymmetry degree of freedom equilibrates more rapidly than shape or $\mathrm{K}$ equilibration [13], it implies that $\mathrm{K}$ equilibration may not occurring in quasi-fission dominant reactions, resulting in a much smaller value of $\mathrm{K}_{0}{ }^{2}$. Experimentally this will result in a larger angular anisotropy deviating considerably from statistical model calculations $[14,15]$.

Almost all systems involving actinide targets had been found to show anomalous anisotropies irrespective of the entrance channel mass asymmetry. This was attributed to orientation dependent quasi-fission [16]. It was argued that for a highly deformed actinide target nucleus quasifission is more probable for a projectile hitting the polar region of the target nuclei rather than the equatorial region.

\subsection{Measurement of Pre-Scission Neutron Multiplicity}

Based on the assumption that the time scales of quasifission and fusion-fission should be different as one (QF) involves only saddle to scission time, whereas the other (FF) contains pre-saddle time in addition to saddle to scission time, it is expected (and observed experimentally [17]) that the onset of quasi-fission at near-barrier energies should bring about a decrease in overall prescission neutron multiplicity.

\subsection{Measurement of FF Mass Distribution}

The variation of the width of the fragment mass distribution with excitation energies has been established as a reliable probe to quasi-fission, particularly at near barrier energies. It is known that statistical fission of the compound nucleus proceeds over a mass symmetric unconditional fission barrier and the experimentally obtained fragment mass distribution is symmetric around the average mass of the target and the projectile and the width (or standard deviation $\sigma_{\mathrm{m}}$ ) of the mass distribution is a smoothly varying function of excitation energy. Quasi fission, on the other hand, proceeds through a mass asymmetric fission barrier. For a mixture of fusionfission and quasi-fission, the mass distribution may still be peaked around the average of the projectile and the target mass, but the width of the mass distribution may get larger. Therefore, if the proportion of the quasi-fission reaction increases with change in the excitation energy, there will be an increase in the width of the mass distribution deviating from the trend. Such an increase in the width of fragment mass distribution with the decrease in energy has been reported recently around the Coulomb barrier [18, 19]. Similar anomalous increase in the width of mass distribution was observed with decrease in beam energy in fusion of systems with the deformed ${ }^{232} \mathrm{Th}$ target [20].

However, identification of the reaction mechanism (particularly QF) is not always unambiguous using the above mentioned experimental probes. A very interesting case in point is the ${ }^{16} \mathrm{O}+{ }^{238} \mathrm{U}$ system, which is a highly fissile, deformed system and therefore is a probable candidate for quasi-fission at near-barrier energies. Here, in fact, probe 3.1 (ER measurement) and probe 3.2 (angular distribution measurement) discussed above, provided contradictory conclusions regarding the presence/absence of QF. Anomalous increase of fission fragment angular anisotropy with respect to the statistical model prediction has been observed for this system at near-barrier energies, which indicated a significant contribution from quasi-fission reaction [21]. By assuming that the effect of quasi-fission is predominant in 
the sub-barrier region, where the orientation of the deformed target projectile system is crucial to determine the fusion trajectory, Hinde et al explained the anomalous energy dependence of the fragment anisotropy for the ${ }^{16} \mathrm{O}$ $+{ }^{238} \mathrm{U}$ system and concluded that there is a quasi-fission transition at sub-barrier energies. On the contrary, the cross sections of the evaporation residues measured for the same system at near and sub-barrier energies were reported [22] to be consistent with the statistical theory, indicating that the contribution from quasi-fission is not significant.

The VECC group measured the fission fragment mass distribution in reaction ${ }^{16} \mathrm{O}+{ }^{238} \mathrm{U}$ [23]. The variation of the variance of the mass distribution as function of energy is shown in Figure 2. The variance is shown to increase anomalously near the Coulomb barrier, thus indicating the onset of quasi-fission. This is consistent with angular anisotropy measurement for this system [21]. However, the other probes, namely, pre-scission neutron multiplicities [23] and ER cross-section [22] show no real deviation from the statistical model prediction. So, it is argued that the orientation dependent quasi-fission sets in at below barrier energies but it's effect may not be detected in ER and pre-scission neutron multiplicities because the total contribution from quasifission reaction is very low as compared to fusion-fission. Hence change in neutron multiplicities and ER crosssection will not be appreciable for unambiguous detection of QF. Thus fission fragment mass distribution is a more sensitive probe to detect quasi-fission especially for lighter systems $\left(\mathrm{Z}_{\mathrm{P}} \mathrm{Z}_{\mathrm{T}} \sim 800\right)$.

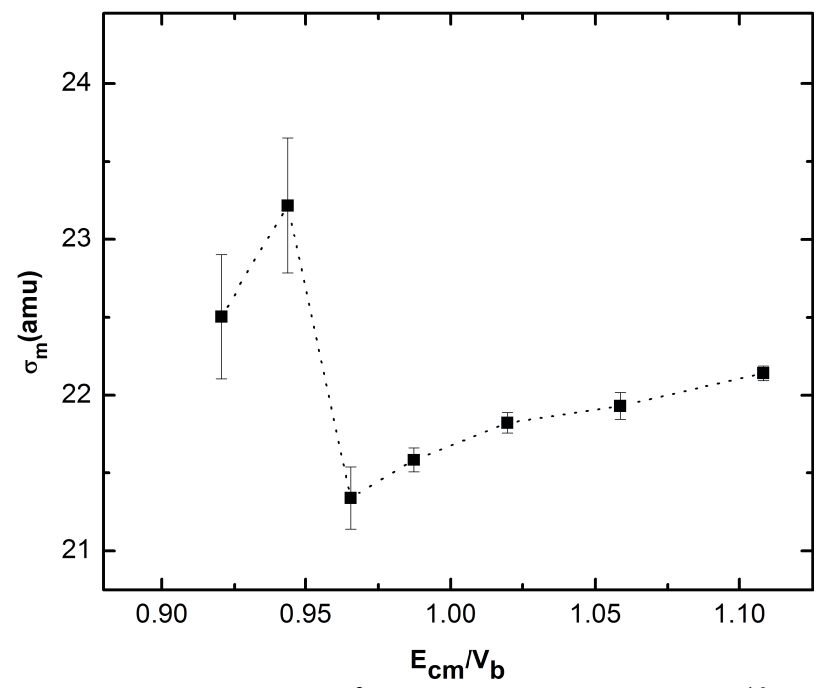

Figure 2. Variation of $\sigma_{\mathrm{m}}{ }^{2}$ with $\mathrm{E}_{\mathrm{CM}} / \mathrm{V}_{\mathrm{B}}$ for the system ${ }^{16} \mathrm{O}+$ ${ }^{238} \mathrm{U}$; the dotted line is to guide the eye.

\section{Experimental study of Quasi-Fission}

The VECC group carried out a series of experiments to understand the dynamics of fusion-fission and quasifission reactions using the accelerator facilities available at Inter University Accelerator Centre (IUAC), New Delhi, and Variable Energy Cyclotron Centre (VECC), Kolkata.
The experiments carried out at IUAC (Table-1) were particularly focused on establishing the role entrance channel effects on quasi-fission. Using pulsed beam from the 15UD Pelletron at IUAC of width $1 \mathrm{~ns}$ and a pulse separation of $250 \mathrm{~ns}$, extensive measurements of mass distribution of fission fragments at near coulomb barrier energies were carried out for different systems.

In the experiment at VECC K130 cyclotron, mass distribution was used as probe to identify the excitation energy at which nuclear shell effect vanishes for actinide nuclei.

Table 1. Summary of reactions studied at IUAC Pelletron

\begin{tabular}{|c|c|c|c|}
\hline System & $\begin{array}{c}\text { Mass } \\
\text { asymmetry } \boldsymbol{\alpha}\end{array}$ & $\begin{array}{c}\text { Ground state } \\
\text { deformation } \\
\boldsymbol{\beta}_{\mathbf{2}}\end{array}$ & $\begin{array}{c}\text { Energy } \\
\text { range } \\
\mathbf{E}_{\mathbf{c m}} / \mathbf{V}_{\mathbf{b}}\end{array}$ \\
\hline${ }^{14} \mathrm{~N}+{ }^{232} \mathrm{Th}$ & $\alpha<\alpha_{\mathrm{BG}}$ & 0.207 & $0.96-1.1$ \\
\hline${ }^{11} \mathrm{~B}+{ }^{235} \mathrm{U}$ & $\alpha>\alpha_{\mathrm{BG}}$ & 0.215 & $0.89-1.27$ \\
\hline${ }^{16} \mathrm{O}+{ }^{238} \mathrm{U}$ & $\alpha<\alpha_{\mathrm{BG}}$ & 0.215 & $0.9-1.12$ \\
\hline${ }^{11} \mathrm{~B}+{ }^{243} \mathrm{Am}$ & $\alpha>\alpha_{\mathrm{BG}}$ & 0.224 & $0.89-1.2$ \\
\hline${ }^{11} \mathrm{~B}+{ }^{232} \mathrm{Th}$ & $\alpha>\alpha_{\mathrm{BG}}$ & 0.207 & $0.92-1.31$ \\
\hline
\end{tabular}

For the detection and effective measurement of flight paths of fission fragments, two indigenously developed MWPCs [24] were used in the experiments. The MWPCs consist of five wire planes; one anode wire plane, two sense wire planes $(\mathrm{X}, \mathrm{Y})$, and two cathode wire planes. The detectors were operated typically at a pressure of about 3 mbar of iso-butane gas. The forward and backward detectors were kept (as shown in Figure 3.) at proper folding angles for two complimentary fission fragments. As folding angle between the fission fragments is an indicator of linear momentum transfer in the reaction, $\mathrm{CN}$

fission events could be distinguished from incomplete fusion processes like transfer fission through folding angle distribution of the fission fragments. The flight times for each event were measured through the fast anode pulse with respect to the pulsed beam. The X-Y position information and energy loss of the fragments in the detector were also recorded through the data acquisition system (DAQ). A Faraday cup and two silicon surface barrier detectors placed at forward angles were used for beam monitoring as well as to normalize the data. 




Figure 3. Photograph of the typical experimental setup. Two large area MWPCs are mounted at the correct folding angle to detect complementary fission fragments.

Masses of fission fragments were extracted from the time of flight differences of the fission fragments [18]. The method is particularly useful, when the beam resolution is not good (particularly for cyclotron).

The systems that were studied using the IUAC pelletron is summarized in the Table 1. All these studies were carried out near the Coulomb barrier energies.

\subsection{Entrance Channel Mass Asymmetry and Quasi-Fission}

To explore the role of entrance channel mass asymmetry on quasi-fission, two reaction pathways were chosen which populate the same compound nuclei. ${ }^{11} \mathrm{~B}$ on ${ }^{235} \mathrm{U}$ and ${ }^{14} \mathrm{~N}$ on ${ }^{232} \mathrm{Th}$ reactions were investigated [25]. The two entrance channels not only populate the same compound nucleus ${ }^{246} \mathrm{Bk}$, but also have similar target deformations and they lie on either side of Businaro Gallone mass asymmetry $\left(\alpha_{\mathrm{BG}}=0.893\right)$. As both the targets have similar $\beta_{2}$ values, any contribution to quasifission from a particular pathway would be solely due to entrance channel mass asymmetry. This is because probability of the initial di-nucleus forming an elongated shape and undergoing orientation dependent quasi-fission is expected to be similar for both the systems.

A system with mass asymmetry $\alpha$ larger than the critical mass asymmetry $\alpha_{\mathrm{BG}}$ will have mass flow from the projectile to the target in the initial di-nucleus, thus establishing a mono-nuclear compact shape which facilitates equilibrium in all degrees of freedom. Therefore quasi-fission is not expected for such system. On the other hand, for a more symmetric mass pair $(\alpha<$ $\alpha_{\mathrm{BG}}$ ) the tendency of mass flow will be towards the projectile from the target, leading to formation of a more symmetric di-nucleus rather than evolving into a mono nucleus; finally, the system will be fissioning through an asymmetric conditional saddle leading to quasi-fission.

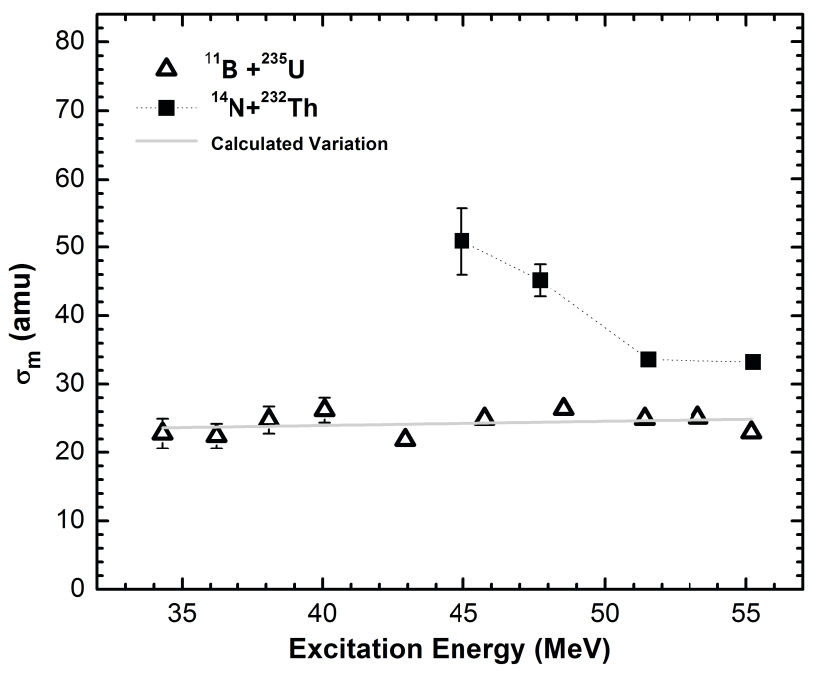

Figure 4. Variation of the width $\left(\sigma_{\mathrm{m}}\right)$ of mass distribution with excitation energy. The solid curve shows the expected $\sigma_{\mathrm{m}}[25]$.

The variation of measured width (standard deviation) of mass distribution with excitation energy is displayed in Figure 4. It is seen that for the more asymmetric system ${ }^{11} \mathrm{~B}+{ }^{235} \mathrm{U}\left(\alpha>\alpha_{\mathrm{BG}}\right)$, the widths of the mass distribution increase linearly with the increase in excitation energy, indicative of fusion-fission process from an equilibrated compound nucleus. However, for the system ${ }^{14} \mathrm{~N}+{ }^{232} \mathrm{Th}$, there is a sudden increase in width of the mass distribution near the Coulomb barrier energies. As the ground state deformation of the two targets are similar (see Table 1), it can be concluded that the anomalous increase of the widths of the mass distribution (or quasifission) in the reaction ${ }^{14} \mathrm{~N}+{ }^{232} \mathrm{Th}\left(\alpha<\alpha_{\mathrm{BG}}\right)$ near the coulomb barrier is primarily due to entrance channel mass asymmetry. Experiments have already been done with another pair of systems $\left({ }^{16} \mathrm{O}+{ }^{238} \mathrm{U}\right.$ and $\left.{ }^{11} \mathrm{~B}+{ }^{243} \mathrm{Am}\right)$ having similar target deformation and populating same compound nucleus to validate the role of entrance channel asymmetry in deciding the fission trajectory. The analysis of the data is in progress.

\subsection{Shell effect and Fragment Mass Distribution}

The study of quasi-fission is considered to be important for finding out the optimum route to synthesis of super heavy elements. While many new SHEs have been artificially synthesized, the existence of SHE is in itself an enigma, considering the fact that according to liquid drop model (LDM) of nucleus as proposed by Niels Bohr [26], element beyond atomic number 104 cannot survive as the fission barrier vanishes. Although, LDM fission barrier vanishes, the addition of shell correction term [27] with LDM energies alters the fission barrier appreciably so as to develop large enough barrier that can increase the fission half-lives by several orders of magnitude. Two kinds of shell-effects on the di-nuclear system have been observed. The first one is due to shell closures in the entrance channel [28], the second one, as studied here in the case of fusion fission, is due to shell-effects in final fragments [29-31]. It is known that with the increase in excitation energy, nuclear shell effect decreases. Earlier 
studies have indicated that the transition (vanishing of shell effect) might occur in the excitation energy range of 40-50 MeV (see [9] for details).

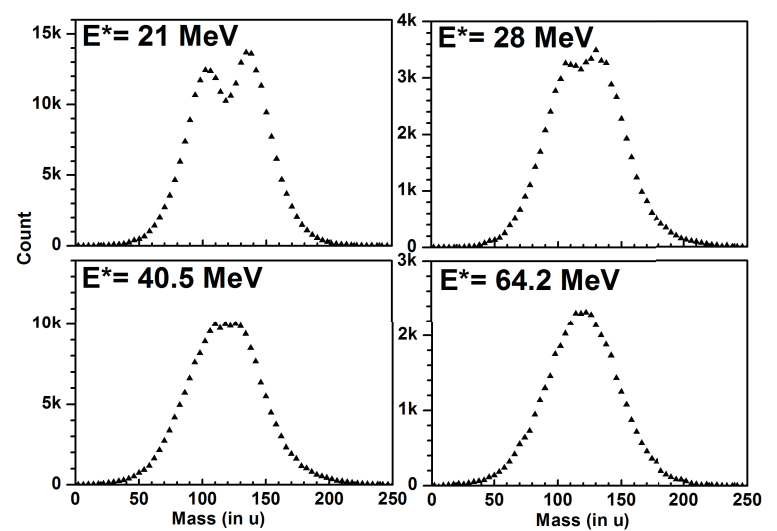

Figure 5. Mass distribution of ${ }^{236} \mathrm{U}$ obtained from the fusion of ${ }^{4} \mathrm{He}$ with ${ }^{232} \mathrm{Th}$ at different excitation energies. The distribution becomes symmetric with increasing excitation energy.

It is well known that the asymmetry in fragment mass distribution in actinide nuclei is a consequence of shell effect in the fissioning nuclei; hence the decrease of contribution of the asymmetric component of the mass distribution with excitation energy would signify weakening of shell effect in the fissioning nucleus. This is has recently been observed in an experiment conducted at K130 cyclotron, VECC spanning a wide range of excitation energy. The ${ }^{4} \mathrm{He}$ beam was bombarded on a ${ }^{232} \mathrm{Th}$ target to populate ${ }^{236} \mathrm{U}$. As this system is highly asymmetric, contribution from incomplete fusion is ruled out. From the measured FF mass distributions (Fig. 5) it can be seen that the mass distributions become symmetric with increasing excitation energy. Since The measurement of the mass distributions have been done at small steps of energy intervals, we can reasonably predict the energy at which the asymmetric component vanishes (or becomes insignificant). Preliminary analysis of the data indicates that such transition occurs at about 40-45 $\mathrm{MeV}$ excitation energy.

\section{Conclusions}

Fission fragment mass distributions have been studied for different target projectile combinations at beam energies close to the Coulomb barrier. Fission fragment mass distribution has been found to be a sensitive probe to detect quasi-fission. The role of entrance channel on the quasi-fission reaction mechanism was explored. It was also shown that the mass distribution can be used to study the shell effect and its variation with excitation energy in nuclei.

The Authors take this opportunity to thank all collaborators, accelerator stuff of IUAC and VECC for successful continuation of this programme over a period of time.

\section{References}

1. S. Hofmann and G. Münzenberg, Rev. Mod. Phys. 72, 733 (2000)

2. Ch. Ngo. Prog. Part. Nucl. Phys. 16, 139 (1986)

3. Yu. Ts. Oganessian et al., J. Phys. G 34, R165 (2007), and earlier references therein

4. W. J. Swiatecki, Phys. Scr. 24, 113 (1981)

5. S. Bjornholm and W. J. Swiatecki, Nucl. Phys. A 391, 471 (1982)

6. A. C. Berriman et al., Nature (London) 413, 144 (2001).

7. G. G. Adamian et al., Phys. Rev. C 68, 034601 (2003)

8. V. Zagrebaev and W. Greiner, J. Phys. G 31, 825 (2005)

9. Y. Aritomo, Phys. Rev. C 80, 064604 (2009)

10. M. Huang et al., Phys. Rev. C 82, 044614 (2010)

11. A. Wakhle et al., Phys. Rev. Lett. 113, 182502 (2014)

12. V. E. Oberacker et al., Phys. Rev. C 90, 054605 (2014)

13. J. Töke et al., Nucl. Phys. A 440, 327 (1985)

14. V. S. Ramamurthy and S. S. Kapoor, Phys. Rev. Lett. 54, 178 (1985)

15. S. Kailas, Phys. Rep. 284, 381 (1997)

16. D.J. Hinde et al., Phys. Rev. Lett. 74, 1295 (1995)

17. L. Donadille et al., Nucl. Phys. A 656, 259 (1999)

18. R. Rafiei et al., Phys. Rev. C 77, 024606 (2008)

19. R. G. Thomas et al., Phys. Rev. C 77, 034610 (2008)

20. T.K. Ghosh et al., Phys. Lett. B 627, 26 (2005)

21. D. J. Hinde et al., Phys. Rev. C 53, 1290 (1996)

22. K. Nishio et al., Phys. Rev. Lett. 93, 162701 (2004)

23. K. Banerjee et al., Phys. Rev. C 83, 024605 (2011)

24. T.K. Ghosh et al., Nucl. Instrum. Phys. Res. A 540, 285 (2005)

25. T.K. Ghosh et al., Phys. Rev. C 79, 054607 (2009)

26. N. Bohr, J. A. Wheeler, Phys. Rev. 56, 426 (1939)

27. V. M. Strutinsky, Nucl. Phys. A. 95, 420 (1967)

28. C. Simenel et al., Phys. Lett. B 710, 607 (2012)

29. M. G. Itkis et al., Nucl. Phys. A 734, 136 (2004)

30. K. Nishio et al., Phys. Rev. C 77, 064607 (2008)

31. E.M. Kozulin et al., Phys. Lett. B 686, 227 (2010) 